\title{
Atom Probe Tomography Studies of the Initiation of Localized Corrosion in Aluminum Alloy 2024
}

\author{
R. Parvizi ${ }^{1}$, R.K.W. Marceau², A.E. Hughes ${ }^{2,3}$, P. Cizek², A.M. Glenn ${ }^{3}$, M.Y. Tan ${ }^{1,2}$ and M. Forsyth ${ }^{2}$ \\ 1. Deakin University, School of Engineering, Geelong, VIC 3216, Australia. \\ 2. Deakin University, Institute for Frontier Materials, Geelong, VIC 3216, Australia. \\ 3. Commonwealth Scientific and Industrial Research Organisation (CSIRO), Division of Materials \\ Science and Engineering, Clayton South, VIC 3169, Australia.
}

Initiation of localized corrosion can compromise the durability of advanced light alloys and challenges the value of these materials in aerospace and automotive applications due to premature, sometimes catastrophic failure. The role of nanostructure in localized corrosion initiation is not well understood, which is due primarily to the difficulty of characterizing both chemistry and structure at the atomic scale. We address this difficulty by taking advantage of the high spatial resolution and chemical sensitivity of atom probe tomography (APT) to reconstruct a three-dimensional perspective of the electrochemical heterogeneities that are responsible for the early stages of corrosion of a typical high-strength aluminum alloy, AA2024 [1].

For the first time, we demonstrate that APT reveals the roles of nano-scale particles and defects in the initiation of aqueous corrosion [2]. We show that localized corrosion originates from preferential oxidation and de-alloying of the $\mathrm{Al}_{20} \mathrm{Mn}_{3} \mathrm{Cu}_{2}$ dispersoid particle and $\mathrm{Al}_{2} \mathrm{CuMg}$ (S-phase) precipitates. Furthermore, we observe that piled-up arrays of dislocations act as rapid diffusion pathways for corrosive species, thus promoting localized corrosion at these crystal defects. The size of the microstructural features characterized in this work bridge corrosion activity at the micrometer scale (often related to constituent intermetallic particles) to the nanoscale, at which chemical heterogeneity can lead to local galvanic cells, where corrosion can initiate or propagate.

To facilitate the examination of the role of dislocation structures in the earliest stages of corrosion, we have chosen a typical high-strength aluminum alloy, drawn AA2024-T3 wire, which has a high density of dislocations together with intermetallic particles. Needle-shape specimens for APT analysis were prepared using a standard two-stage electropolishing method, with a $10 \mathrm{vol} \%$ solution of perchloric acid in undiluted ('glacial') acetic acid in the first (rough polishing) stage and a 2 vol.\% solution of perchloric acid in 2-butoxyethanol in the second (fine polishing) stage. For corrosion experiments, the sample tip was immersed in a droplet of aerated distilled water for 5 min to allow free aqueous corrosion and then dried with compressed air. APT experiments were then performed using a LEAP 4000 HR instrument (CAMECA Instruments) operating in voltage mode under ultra-high vacuum $(<2 \times 10-11$ Torr) with the following run parameters: set-point temperature of $20 \mathrm{~K}$, pulse fraction of $15 \%$, pulse repetition rate of $200 \mathrm{kHz}$ and detection rate of 0.005 atoms per pulse.

Finally, the utility of APT to serve as an extremely useful tool for mapping the atomic hydrogen distribution to identify preferred sinks and hydrogen traps is highlighted in the context of corrosion research. Whilst it is recognized that the source of hydrogen in APT experiments is not necessarily the same as in a corrosion experiment (due to the likely sorption of hydrogen gas residual in the atom probe instrument vacuum chamber), the nature of the microstructural adsorption sites will be the same [3]. 


\section{References:}

[1] R Parvizi et al., Langmuir 30 (2014) p. 14817.

[2] R Parvizi et al., Corrosion Science 116 (2017), p. 98.

[3] RKW Marceau, Materials Science and Technology 32 (2016), p. 209. 